\title{
Propiedades físicas y químicas de las grasas bovinas fraccionadas e interesterificadas.
}

\author{
Por M. A. Grompone
}

Facultad de Química, Casilla de Correo 1157,

Montevideo - URUGUAY

\section{RESUMEN}

Propiedades fisicas y quimicas de las grasas bovinas fraccionadas e interesterificadas.

Se determinan la composición en ácidos grasos y las propiedades físicas (punto de fusión, índice de grasa sólida, título) de las fracciones separadas industrialmente por enfriamiento controlado en seco de la grasa vacuna uruguaya. También se estudian los productos obtenidos en el laboratorio por transesterificación de dichas fracciones y por interesterificación de las mismas con diferentes cantidades de aceite de girasol.

En base a este estudio, se dispone de un vasto panorama de los posibles productos derivados de la grasa vacuna y sus aplicaciones. La elección en cada caso entre los distintos productos con características fisicoquímicas similares, dependerá también de factores económicos (costo de materias primas, de operaciones, etc) y técnicos (estabilidad frente al enranciamiento, etc).

PALABRAS-CLAVE: Fraccionamiento térmico - Grasa vacuna - Interesterificación - Transesterificación.

\section{SUMMARY}

Physical and chemical properties of fractionated and interesterified beef tallows.

Fatty acid composition and physical properties (melting point, solid fat index, titer) of the fractions obtained from the Uruguayan beef tallow by industrial dry cooling process were determined. Products obtained on the laboratory by transesterification of these fractions and interesterification of them with sunflower oil were also studied.

With these results it is better understanding their behaviour and potential end-use applications. The selection among different products with similar physicochemical properties depends not only upon economical factors (raw material cost, operation costs, etc.) but technical (stability to rancidity, etc.) as well.

KEY-WORDS: Beef fat - Interesterification - Thermic fractionation - Transesterification.

\section{INTRODUCCION}

La grasa vacuna tiene un rango plástico restringido y un punto de fusión elevado por lo que no es adecuada para alimentos de buena calidad.
Esto es más crítico para las grasas vacunas uruguayas cuyos puntos de fusión $\left(45.0-48.8^{\circ} \mathrm{C}\right)$ son más altos que los indicados en la bibliografía para las de otros orígenes (1). Generalmente se fraccionan para obtener productos de textura y comportamiento térmico adecuados. Sin embargo, las publicaciones sobre el fraccionamiento de grasas vacunas no son abundantes ni sistemáticas como para brindar un panorama completo de las aplicaciones y posibilidades de dichos productos $(2-9)$.

La transesterificación con catalizadores químicos es una reacción muy estudiada (10), por medio de la cual se desordenan los ácidos grasos que forman los triglicéridos hacia distribuciones al azar. Como las grasas y los aceites naturales poseen un cierto grado de ordenamiento propio, la transesterificación puede producir cambios en sus propiedades físicas (punto de fusión, curva dilatométrica, etc.) que conviene conocer de antemano para predecir la aplicabilidad en cada caso. La bibliografía indica que la transesterificación modifica muy poco los puntos de fusión de las grasas vacunas puras (11) (12) aunque su interesterificación con aceites vegetales produce cambios importantes (13) (14). A pesar de esto, la información sobre la transesterificación de fracciones obtenidas a partir de grasa vacuna o sobre la interesterificación de éstas con otros aceites y grasas no es frecuente. El interés de estos estudios radica no sólo en mejorar la calidad de los productos finales sino también en encontrar aplicaciones para subproductos de uso limitado, tales como las oleoestearinas.

En este trabajo se estudian las propiedades de las fracciones obtenidas por enfriamiento controlado, en seco, de grasa vacuna uruguaya, las de los productos obtenidos por su transesterificación y las de los obtenidos por su interesterificación con aceite de girasol. 


\section{PARTE EXPERIMENTAL}

En todos los ensayos se utilizaron productos obtenidos industrialmente por fraccionamiento térmico de grasas vacunas uruguayas, de acuerdo con el diagrama esquematizado en la Figura 1. Se incluye en él el promedio de los rendimientos para cada operación y los rangos de variación de los puntos de fusión y de los títulos para cada fase. Dichos productos fueron transesterificados en el laboratorio de acuerdo con la técnica descrita en la literatura (12): el material graso, adicionado de $0,5 \%$ de solución de $\mathrm{NaOH}$ al $50 \%$ y de $1 \%$ de glicerol al $95 \%$ (catalizadores), se mantuvo a $60^{\circ} \mathrm{C}$ bajo vacío hasta eliminar totalmente el agua y luego se calentó a $120^{\circ} \mathrm{C}$ durante 30 minutos (manteniendo el vacío) para llevar a cabo la reacción. También se interesterificaron muestras de grasa vacuna o de oleoestearina (obtenida industrialmente por fraccionamiento térmico en seco) con aceite de girasol, según el método anteriormente indicado.

OLEOOLEINA OO-42-37

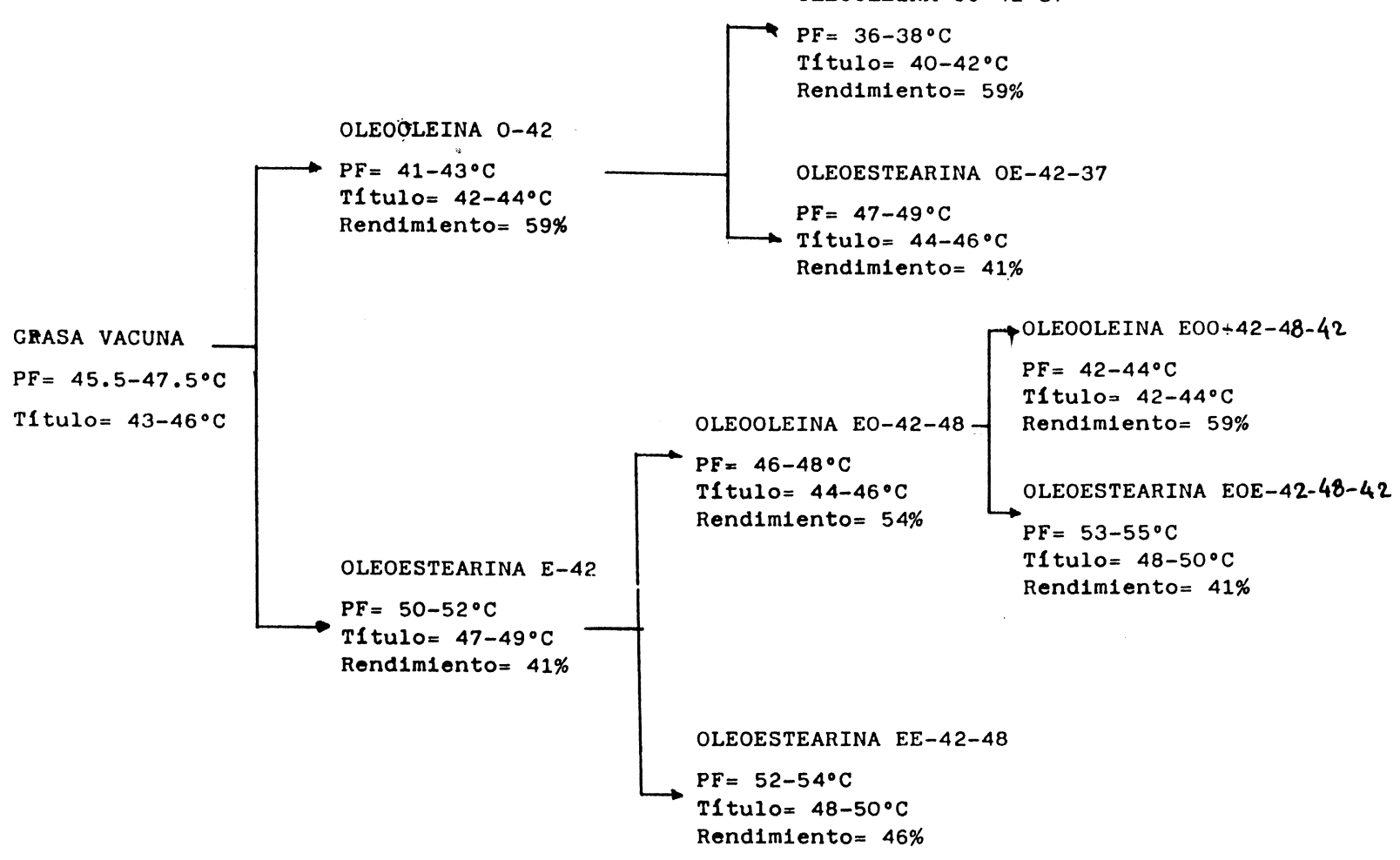

Figura 1

Tanto las materias primas como los productos finales se analizaron de acuerdo con las técnicas oficiales de la American Oil Chemists'Society (15):

1.- Los ésteres metílicos de los ácidos grasos se analizaron según los métodos Ce 2-66 y Ce 1-62, en un cromatógrafo de gases marca Shimadzu modelo GC-6, equipado con columnas de SP-2330 al $10 \%$ sobre Supelcoport (100/120 mallas), de acero inoxidable, de 10 pies de longitud y $1 / 8$ de pulgada de diámetro. Se usó nitrógeno como gas portador, detector de ionización de llama y $185^{\circ} \mathrm{C}$ como temperatura de columnas.

2.- Título según el método $\mathrm{Cc}$ 12-59.

3.- Punto de fusión (capilar abierto) según el método Cc 3-25.

4.- Indice de grasa sólida según el método $\mathrm{Cd}$ 10-57.

\section{RESULTADOS}

Las propiedades fisicoquímicas se estudiaron en función de las materias primas empleadas: 


\subsection{Grasa vacuna fraccionada.}

La composición en los ácidos grasos mayoritarios (palmítico, esteárico y oleico) de las fracciones obtenidas industrialmente, se resume en la
Figura 2 mediante un diagrama de barras. Las fracciones se ordenan de acuerdo con porcentajes crecientes de ácido oleico; de esta manera resultan valores aproximadamente decrecientes en los porcentajes de los ácidos palmítico y esteárico.

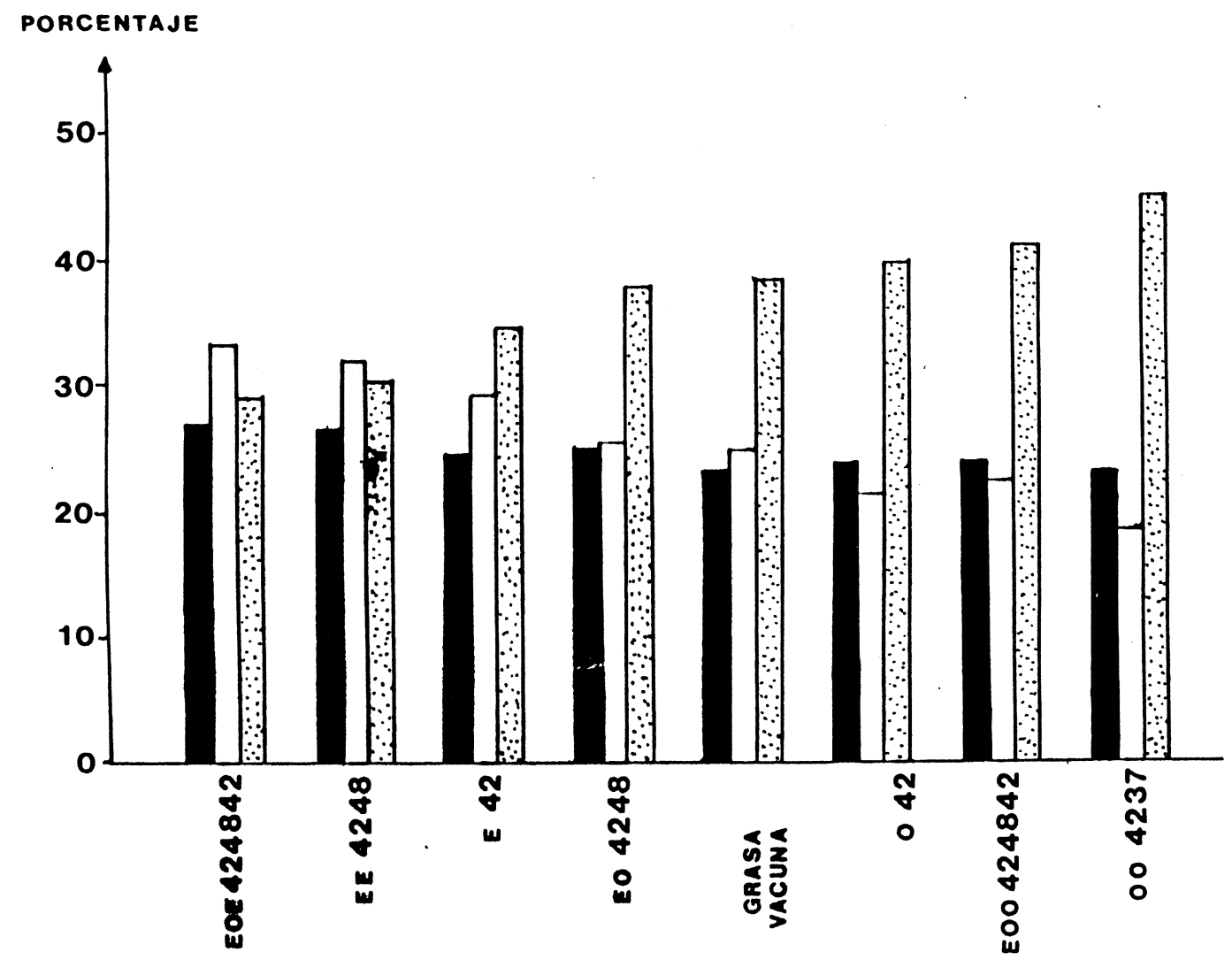

Figura 2

Composición en los ácidos grasos mayoritarios de las fracciones obtenidas industrialmente a partir de la grasa vacuna.

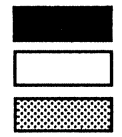

Acido palmítico.

Acido esteárico.

Acido oleico.

En la Figura 3 se muestran las curvas dilatométricas para la grasa vacuna de partida y para tres de las fracciones obtenidas a partir de ella $(E-$ $42,0-42$ y 00-42-37). Se observa que las curvas no modifican sensiblemente su forma aunque se desplazan hacia valores menores a medida que disminuye el punto de fusión.

\subsection{Grasa vacuna fraccionada y transeste-} rificada.

En la Figura 4 se grafican los puntos de fusión de las muestras transesterificadas en función de los puntos de fusión de las muestras originales. Para valores alrededor de $42^{\circ} \mathrm{C}$, la transesterificación no modifica el punto de fusión. Para valores menores, la transesterificación aumenta el punto de fusión y para valores mayores, lo disminuye. Como las grasas vacunas uruguayas tienen puntos de fusión comprendidos en el rango $45,5-47,5^{\circ} \mathrm{C}$, la transesterificación baja el punto de fusión, mejorando su calidad. Lo mismo sucede con las fases "mas duras" (oleoestearinas) obtenidas a partir de ella. Para las fases "más blandas" (oleooleínas) este procedimiento no es útil ya que provoca un aumento en el punto de fusión.

En la Figura 5 se muestran las curvas dilatométricas para la grasa vacuna original y la transesterificada y para la oleooleína 00-42-37 original y transesterificada. Se nota un comportamiento 


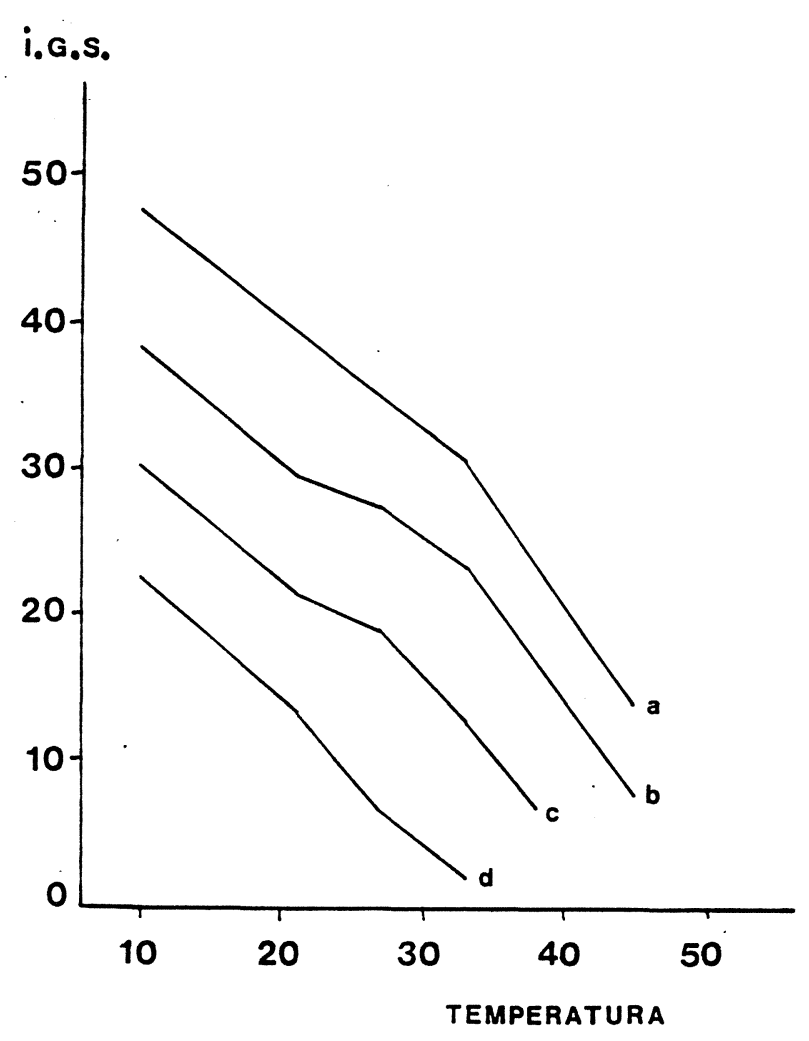

Figura 3

Indice de la grasa sólida (I.G.S.) en función de la temperatura para la grasa vacuna (b) y para las fracciones E-42 (a), 0-42 (c) y 00-42-37 (d).

\section{PF(final)}

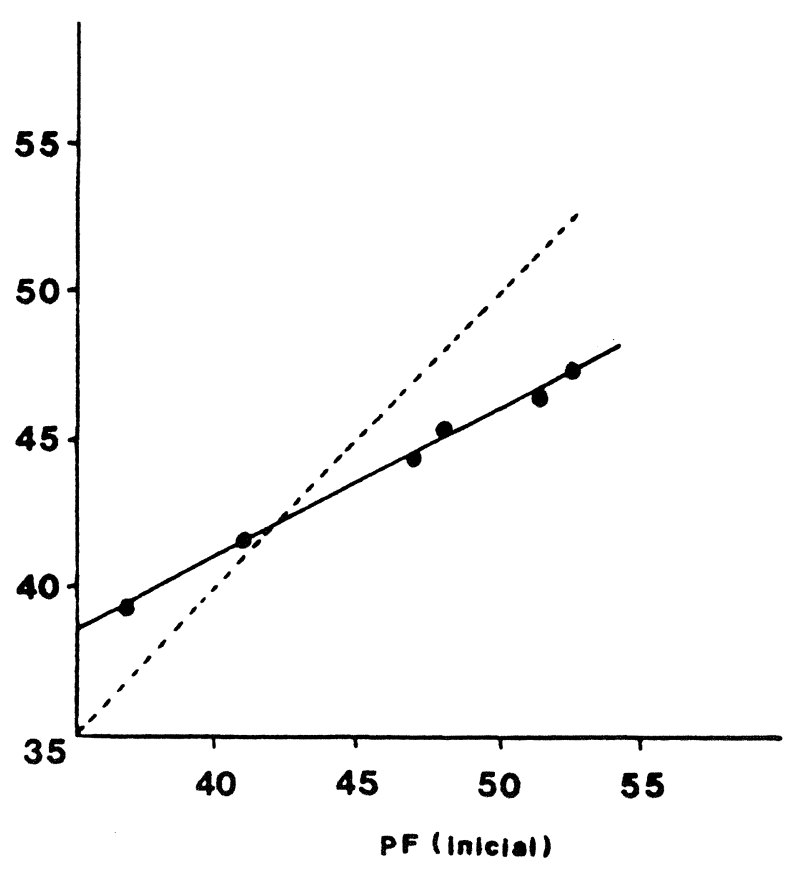

Figura 4

Puntos de fusión de muestras transesterificadas en función de los puntos de fusión de las muestras iniciales.

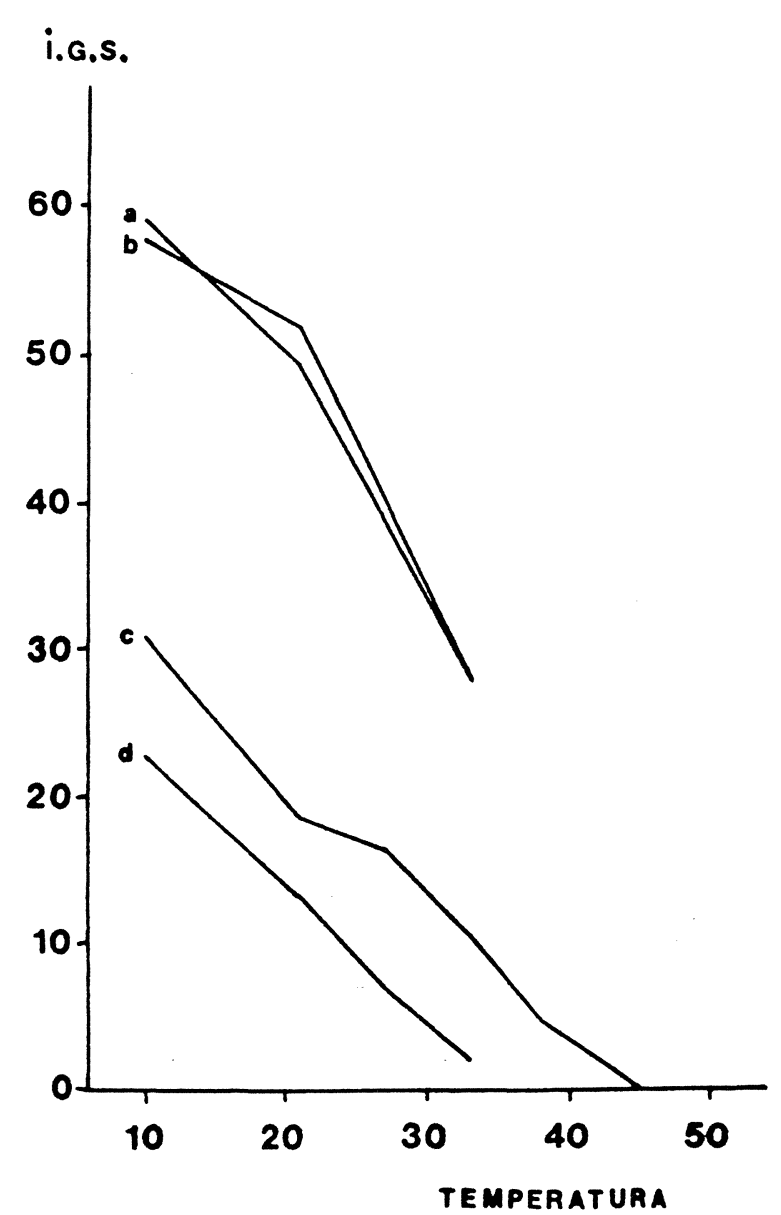

Figura 5

Indice de grasa sólida (I.G.S.) en función de la temperatura para la grasa vacuna transesterificada (a) e inicial (b), y para la fracción 00-42-37 transesterificada (c) e inicial (d).

similar al del punto de fusión: en la grasa vacuna prácticamente no se modifica la curva dilatométrica y en la oleooleína, la desplaza hacia valores más altos.

En la Figura 6 se grafica el punto de fusión de las muestras originales y de las transesterificadas en función del título. De acuerdo con (9), las grasas vacunas y las oleoestearinas tienen puntos de fusión mayores que sus respectivos títulos, y las oleooleínas, menores. Sin embargo, todas las muestras transesterificadas tienen puntos de fusión muy cercanos a sus respectivos títulos (aunque generalmente menores).

La oleoestearina EE-42-48, con punto de fusión $52.8^{\circ} \mathrm{C}$ y con título $48.0^{\circ} \mathrm{C}$, transesterificada presenta un punto de fusión de $47.6^{\circ} \mathrm{C}$. Si dicho producto transesterificado se fracciona a la tem- 


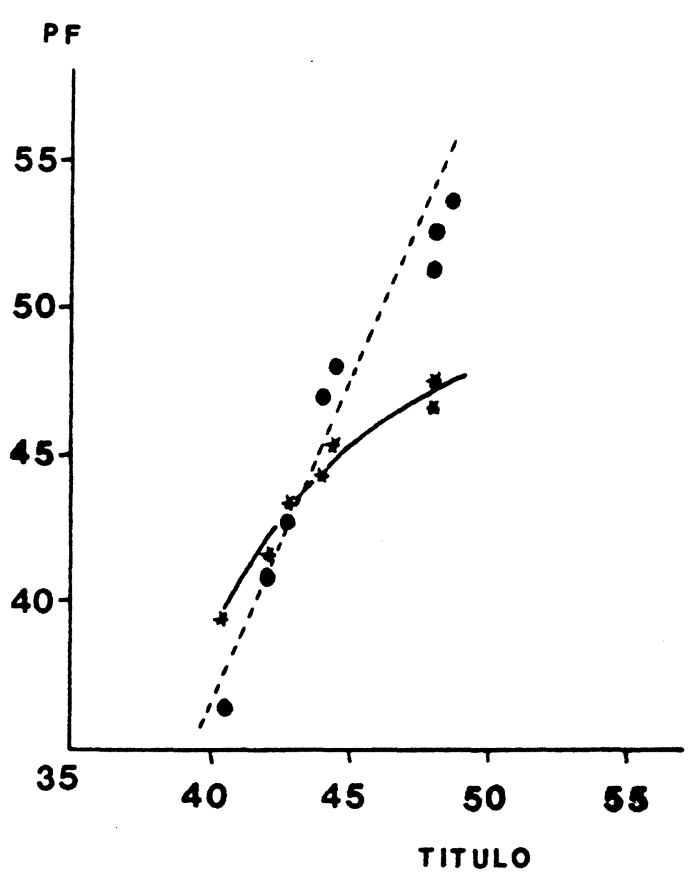

Figura 6

Punto de fusión de las muestras iniciales $(\bullet)$ y de las transesterificadas $\left(^{*}\right)$ en función del título.

peratura de $36^{\circ} \mathrm{C}$, se obtiene una fase líquida de punto de fusión $44.8^{\circ} \mathrm{C}$ (con un rendimiento del $45 \%$ ) y una fase sólida con un punto de fusión de $49,6^{\circ} \mathrm{C}$. La composición de la fase sólida es similar a la de la fracción EE-42-48 (obtenida industrialmente) que tiene un punto de fusión comprendido en el rango $52-54^{\circ} \mathrm{C}$ y a la fracción EOE-42-48-42 (también obtenida industrialmente) que tiene un punto de fusión comprendido en el rango $53-55^{\circ} \mathrm{C}$. La composición de la fase líquida es parecida a la de la fracción E-42 (obtenida industrialmente) que tiene un punto de fusión comprendido en el rango $50-52^{\circ} \mathrm{C}$. Sin embargo, su punto de fusión es considerablemente menor $\left(44.8^{\circ} \mathrm{C}\right)$. Esto enfatiza el hecho de que no alcanza con conocer la composición en ácidos grasos para predecir el comportamiento físico.

\subsection{Mezclas de grasa vacuna o de sus frac-} ciones con aceite de girasol.

En la Figura 7 se grafica el punto de fusión de las mezclas de grasa vacuna y aceite de girasol, en función del contenido en aceite.

En la Figura 8 se construye la misma gráfica para mezclas de oleoestearina (del tipo EE-42-48) y aceite de girasol. En la Figura 9 se grafica el punto de fusión de las mezclas interesterificadas de grasa vacuna u oleoestearina con aceite de girasol, en función del punto de fusión de la mezcla original correspondiente. En las figuras 10 y 11 se indican las curvas dilatométricas para dos mezclas, antes y después de la interesterificación.

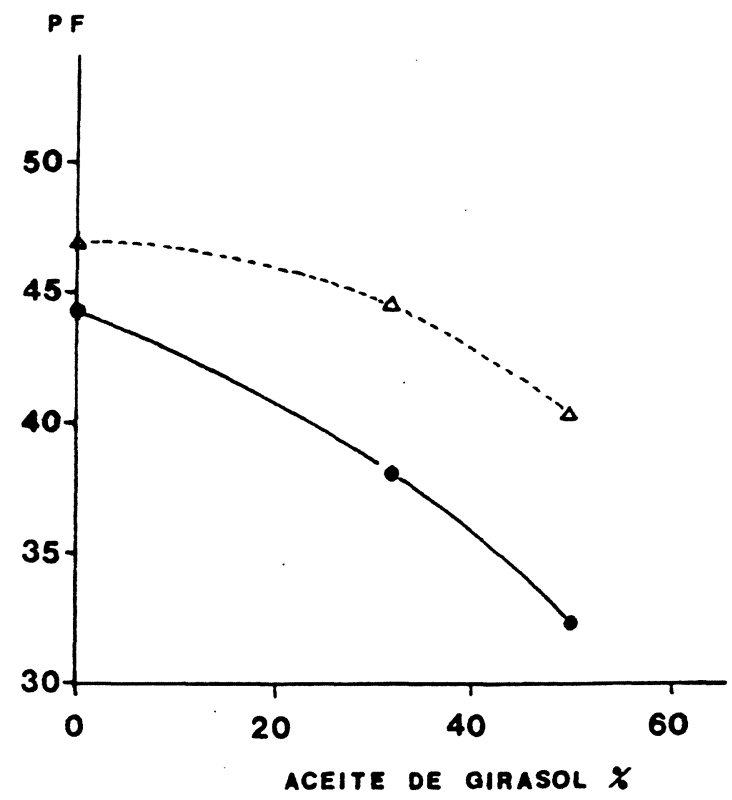

Figura 7

Punto de fusión de las mezclas de grasa vacuna y aceite de girasol en función de su contenido en aceite.

$\Delta$ muestra inicial. $\quad-$ muestra interesterificada.

Se concluye que la interesterificación disminuye apreciablemente los puntos de fusión de las mezclas, tanto más cuanto más aceite contengan. Este efecto es más pronunciado con la oleoestearina que con la grasa vacuna. Una mezcla interesterificada de $67 \%$ de grasa vacuna y $33 \%$ de aceite de girasol presenta un punto de fusión $\left(38.0^{\circ} \mathrm{C}\right)$ igual al de la oleooleína 00-42-37. Si se comparan sus curvas dilatométricas resulta un poco menos plástica la oleooleína. Por lo tanto, la elección entre esos dos tipos de productos depende de manera considerable de otros factores: estabilidad a la oxidación, costo de las materias primas y de las operaciones, etc.

La oleoestearina interesterificada con aceite de girasol en cantidades comprendidas entre $20 \%$ y $33 \%$ de productos cuyos puntos de fusión caen prácticamente todos en el rango de la oleooleína $0-42$. Esto significa que la elección entre ambos tipos de productos también depende de otros factores, como en el caso anteriormente señalado. 


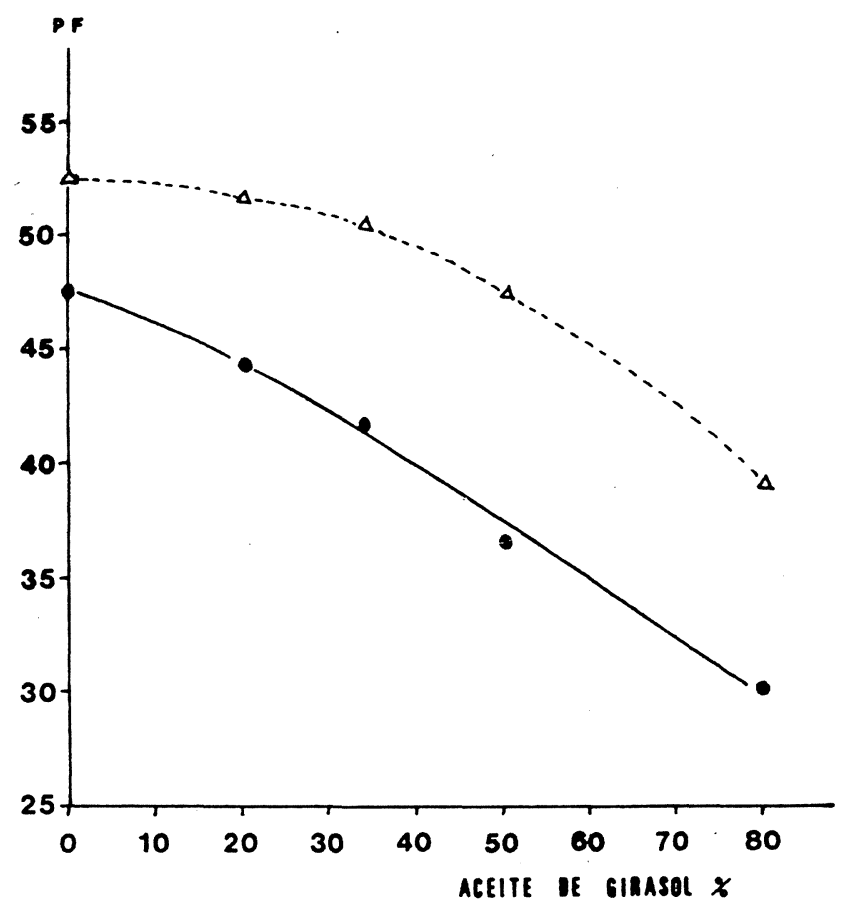

Figura 8

Punto de fusión de las mezclas de oleoestearina (del tipo EE-42-48) y aceite de girasol en función del contenido en aceite.

$\Delta$ muestra inicial. $\quad$ muestra interesterificada.

\section{PF(final)}

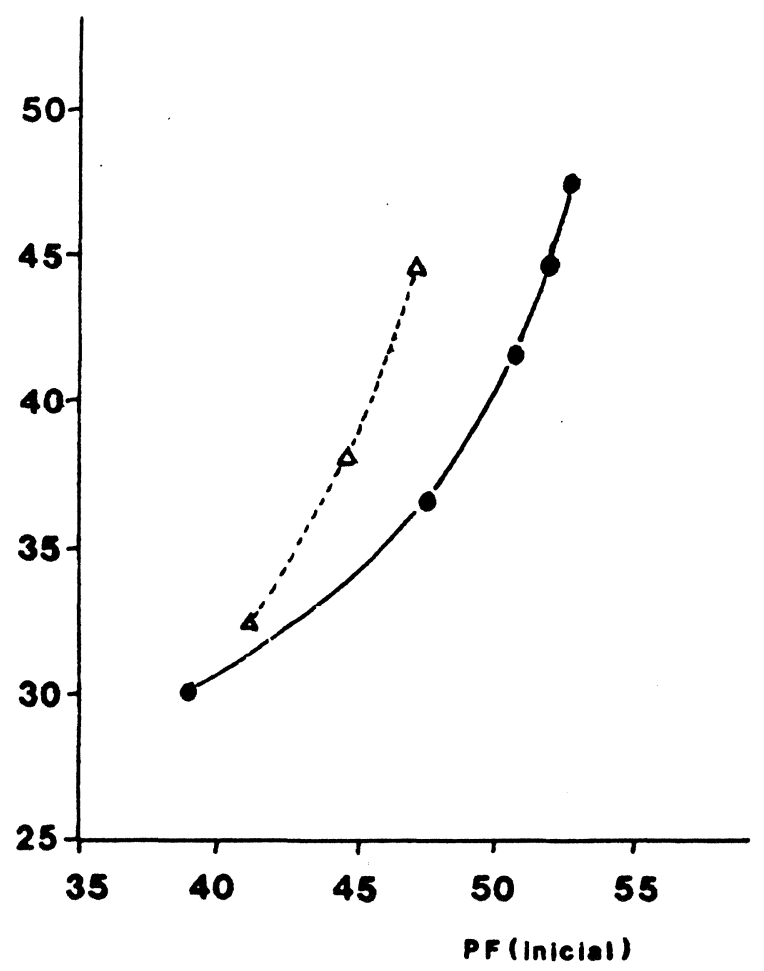

Figura 9

Punto de fusión de mezclas interesterificadas en función del punto de fusión inicial.

- mezcla de oleoestearina EE-42-48 y aceite de girasol.

$\Delta$ mezcla de grasa vacuna y aceite de girasol.

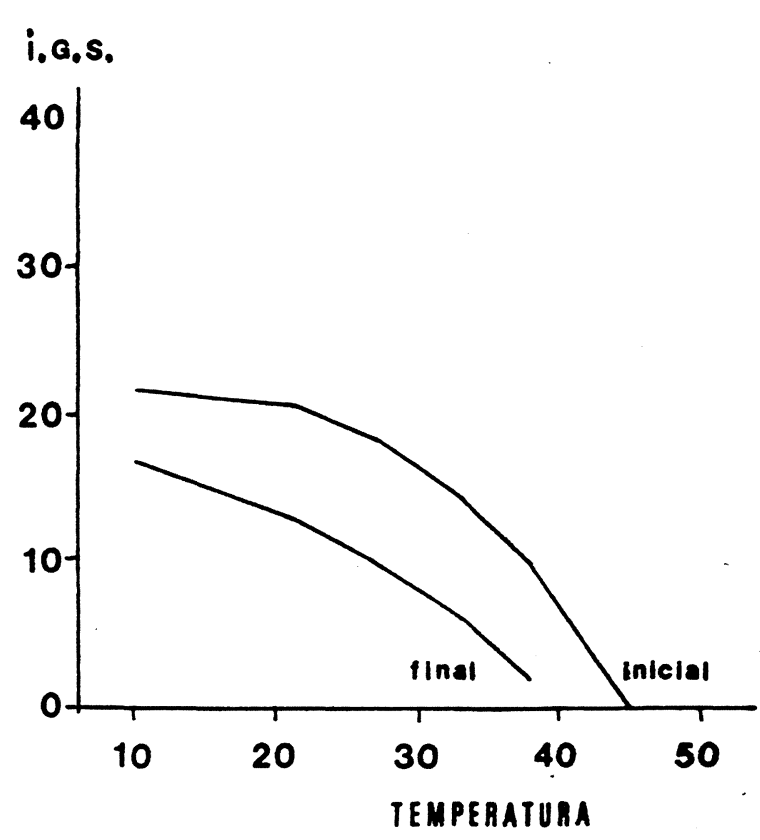

Figura 10

Indice de grasa sólida (I.G.S.) en función de la temperatura para la mezcla inicial e interesterificada de $2 / 3$ de grasa vacuna y $1 / 3$ de aceite de girasol.

i.G.s.

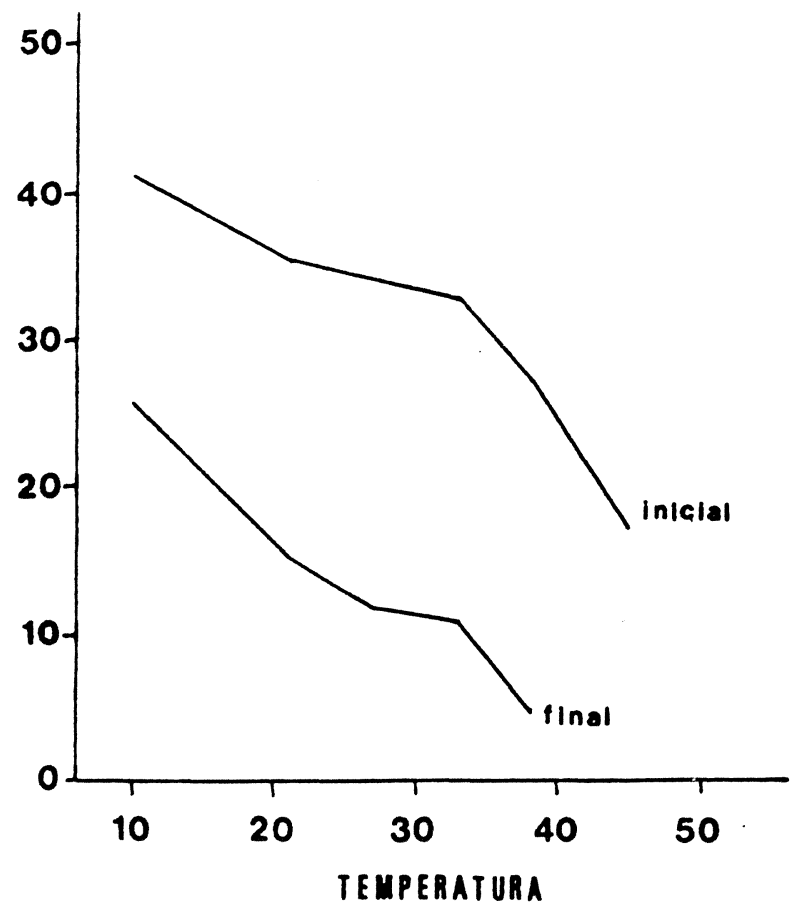

Figura 11

Indice de grasa sólida (I.G.S.) en función de la temperatura para la mezcla inicial e interesterificada de $2 / 3$ de oleoestearina EE-42-48 y $1 / 3$ de aceite de girasol. 


\section{CONCLUSIONES}

Las grasas vacunas suelen modificarse para obtener productos "más blandos". Esto se puede lograr ya sea por fraccionamiento térmico, ya sea por interesterificación con aceites vegetales.

La obtención de oleooleínas por fraccionamiento térmico de grasas vacunas implica necesariamente la producción de oleoestearinas. Estas no tienen usos específicos ni amplios y es deseable modificar sus propiedades físicas para obtener productos de mejor calidad y mayor valor comercial, lo que se puede lograr por diferentes vías: a) fraccionamientos sucesivos que den origen a fases con propiedades físicas especiales; b) hidrogenación parcial para obtener otras oleoestearinas de menor índice de yodo que tengan empleo en otras industrias; c) interesterificación con aceites vegetales que conduzcan a productos con puntos de fusión menores, más fáciles de utilizar en el área de las grasas comestibles (la simple mezcla de ambos separa fases fácilmente debido a que sus componentes presentan puntos de fusión demasiado alejados); d) transesterificación para disminuir su punto de fusión a valores comparables con los del material de partida.

La elección del proceso a llevar a cabo, una vez determinadas las características fisicoquímicas de los productos obtenidos en cada caso, supone fundamentalmente el estudio económico del mismo, en el cual habrá que tener en cuenta, entre otros, el costo de las materias primas, el costo de las operaciones y las pérdidas en productos secundarios.

\section{BIBLIOGRAFIA.}

1. Grompone, M.A. y Moyna, P.- "Characteristics of Uruguayan Beef Tallown.- J. Am. Oil Chemists' Soc. 60 (1983) 1331-1332

2. Luddy, F.E. Hampson, J.W. Herb, S.F. y Rothbart H.L."Development of edible tallow fractions for specialty fat uses".- J. Am. Oil, Chemists'Soc. 50 (1973) 240-244
3. Taylor H.H. Luddy, F.E. Hampson J.W. y Rothbart, H.L."Substitutability of fractionated beef tallow for other fats and oils in the food and confectionary industries: an economic evaluation".- J.Am. Oil Chemists'Soc. 53 (1976) 491-495

4. Bussey, D.M Ryan, T.C. Gray, J.I. y Zabik, M.E.- "Fractionation and characterization of edible tallow".- J. Food Sci. 46 (1981) $526-530$

5. Kozempel, M.F. Craig, J.C. Heiland, W.K. Elias, S. y Aceto, N. C.-"Development of a continuous process to obtain a confectionery fat from tallow: final status".- J. Am. Oil Chemists'Soc. 58 (1981) 921-925.

6. Rastoin, J.- "Traitement des corps gras: Hydrogénation. Fractionnement. Interestérification".- Rev. Fr. Corps Gras 32 (1985) 97-102

7. Sonnet, P.E. Pfeffer, P.E. y Wise, W.B.- "Evaluation of some approaches to liquified tallow: stereochemical consequences of inter-esterification".- J. Am. Oil Chemists'Soc. 63 (1986) 1560-1564

8. Bernardi, C.M. Freyre M.R. y Garoni, O.- "Estabilidad de fracciones de grasa bovinan- Grasas y Aceites 39 (1988) 367-372

9. Grompone, M.A.- "Physicochemical properties of fractionated beef tallows".- J. Am. Oil Chemists'Soc. 66 (1989) 253255

10. Swern, D. (Editor).- "Bailey's industrial oil and fat products".Vol. 2 p.147-164.- 4a Edición, J. Wiley and Sons, New York, 1979

11. Luddy, F.E. Morris, S.G. Magidman, P. y Riemenschneider, R.W.-"Effect of catalytic treatment with sodium methylate on glyceride composition and properties of lard and tallow.- J. Am. Oil Chemists'Soc. 32 (1955) 522-525

12. Sreenivasan, B.- "Interesterification of fats".- J. Am. Oil Chemists'Soc. 55 (1978) 796-805

13. Chobanov, D. y Chobanova, R.- "Alterations in glyceride composition during interesterification of mixtures of sunflower oil with lard and tallown- J. Am. Oil Chemists'Soc 54 (1977) $47-50$

14. Lo, Y.C. y Handel, A.P.- "Physical and chemical properties of randomly interesterified blends of soybean oil and tallow for use as margarine oils".- J. Am. Oil Chemists'Soc. 60 (1983) $815-818$

15. Official and tentative methods of the American Oil Chemists'Society.- R.C. Walker (Editor).- A.O.C.S., Champaign, IL, 1986

(Recibido: Noviembre 1990) 